\title{
SENSORS AND ACTUATORS, TWENTE
}

\section{P. BERGVELD}

Faculty of Electrical Engineering, University of Twente, Box 217 , Enschede (The Netherlands)

\begin{abstract}
This paper describes the organization and the research programme of the Sensor and Actuator (S\&A) Research Unit of the University of Twente, Enschede, the Netherlands. It includes short descriptions of all present projects concerning: micromachined mechanical sensors and actuators, optical sensors, recording media and sensors based on magnetic materials, FET-based sensors and systems and the integration of electronic functions and systems in sensor chips.
\end{abstract}

\section{Introduction}

The University of Twente was officially founded in 1961 as the first Dutch campus University, starting with Faculties of Electrical, Mechanical and Chemical Engineering. At present, the University of Twente accommodates ten faculties of technical as well as social sciences, at which 6000 students are studying.

From the beginning, the disciplinary groups in the Faculty of Electrical Engineering were chosen around information technology and several researchers started sensor activities. The first publications on the development of ISFETs [1, 2] and magnetoresistors [3] are well known, followed by more and more original papers on different types of sensors. These individual initiatives were coordinated by the establishment of the Sensor \& Actuator Research Unit in 1981, regulated by law as a strategy for promot. ing interdisciplinary activities. Funds were raised to fit up a special Sensor and Actuator Laboratory for combined use of IC and thin-film facilities, which at present operates for about 60 regular group members of the Faculties of Electrical Engineering, Applied Physics and Chemical Engineering. In this way, a wide range of different disciplines are linked together as shown in Fig. 1.

The location of the Sensor and Actuator Laboratory is in the building shared by the Faculties of Electrical Engineering and Applied Physics; it is planned to be renewed and enlarged in the coming years as a separate clean-room facility for the Sensor \& Actuator Research Unit and the IC Technology Group. 


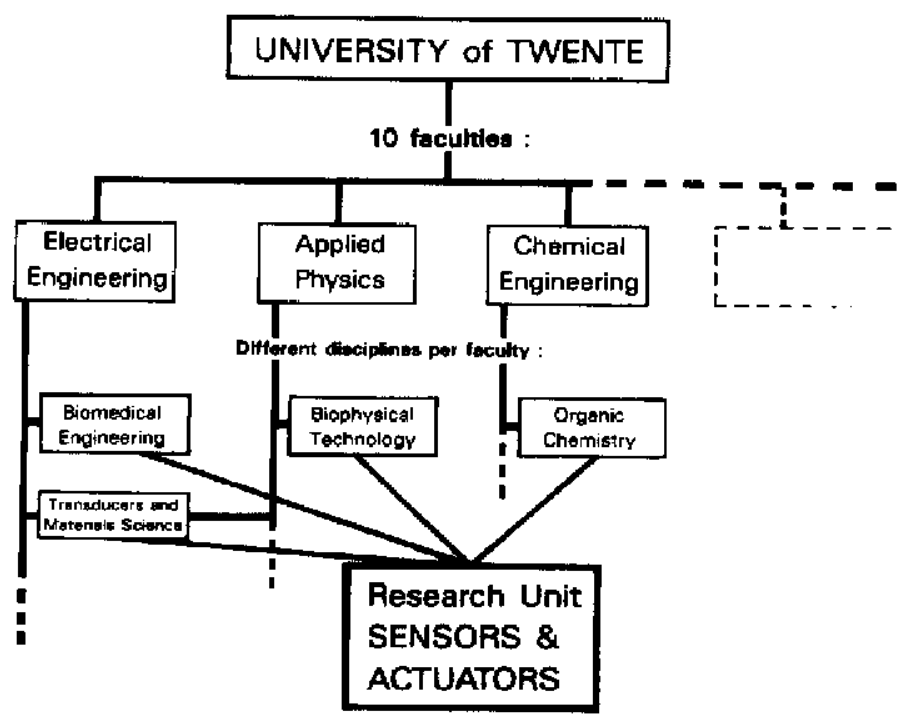

Fig. 1. Schematic representation of Sensors \& Actuators Research Unit organization at the University of Twente, Enschede, the Netherlands.

In 1986 the Ministries of Science and Education and of Economic Affairs decided to stimulate Twente's initiatives with funding of a large amount of modern equipment to be installed in the new clean-room location, making it into the official national sensors and actuators centre.

\section{Organization}

The Twente Sensor and Actuator Research Unit is directed by chosen members of the collaborating disciplines, with the programmatic coordination and stimulation of the different research projects being the main task. The S\&A laboratory is managed by the head of the laboratory, guided by a separate group of members chosen from the different disciplines, whose special task is the definition of technologies and equipment that can be used by as many members as possible.

From the beginning, a self-service operation was chosen for the S\&A laboratory, forcing the individual member researchers and Ph.D. as well as M.Sc. students to make not only their own designs, but also to realize the prototypes. In this way, direct feedback between final experimental results and original design goal, including technological realization should be encouraged. The relatively small staff of the laboratory maintains all facilities, installs new equipment and trains the members of the research unit to become familiar with the different technologies and processes.

Research program

The initial research programme of the S\&A Research Unit was, of course, the sum of the original research topics of the collaborating disciplines, but over the years an increasing number of disciplines of different faculties have became interested in S\&A research. Now the various activities 
are more connected, leading to an intrinsic multidisciplinary approach of the Twente S\&A group as a whole.

The research projects are partly devoted to fundamental material science, related compositionally to the various technologies for depositing thin layers, with their specific microstructural and physical properties. Other research projects are exploiting this fundamental knowledge in the construction of novel sensors for a large variety of applications.

The main topic is the development of micro devices, partly based on well-known physical principles that can be better exploited on a microscale than in the original more mechanical way of construction, and partly based on new principles or methods, made possible due to the smaller dimensions that can be achieved with modern micro technologies. In both cases, advantage is taken of the multiscale production possibilities, known from IC technology, as well as the possibility of integration of functions. The various present projects can therefore be grouped together with respect to the following specialities:

(a) micromachined mechanical sensors and actuators;

(b) optical sensors;

(c) recording media and sensors based on magnetic materials;

(d) FET-based sensors and systems;

(e) integration of electronic functions and systems.

This subdivision of research topics will be the guideline for the further exposition of the Twente S\&A activities.

The S\&A laboratory

To support the research activities described above, the S\&A laboratory accommodates the following facilities:

(a) A laboratory for the deposition of thin films by means of evaporation, chemical vapour deposition, sputtering or aerosol deposition.

(b) A photolithographic facility for defining microstructures.

(c) An NMOS production line for the fabrication of FET-based sensors.

(d) Facilities for physical and electrical characterization of thin films and devices.

Furthermore, use can be made of a CMOS production line, including ion-implantation facilities, from the microelectronics and IC technology group of the Faculty of Electrical Engineering. The mask production is carried out in a cooperative mask centre.

Micromachined mechanical sensors and actuators based on anisotropic etching of silicon

The anisotropic etching of silicon is developed for the micromachining of silicon wafers. Originally the desired cavities, holes and channels were etched in a solution of ethylene diamine, pyrocatechol and pyrazine in water (EDP), but at present the micromachining is exclusively carried out in 
a potassium-hydroxide-isopropanol-water solution $(\mathrm{KOH})$ at about $75{ }^{\circ} \mathrm{C}$, resulting in an etch rate of $40 \mu \mathrm{m} / \mathrm{h}$. Usually a $1 \mu \mathrm{m}$ layer of $\mathrm{SiO}_{2}$ acts as an etch mask. Silicon membranes are constructed in this way, the thickness being controlled by monitoring the colour of the diaphragm with transmitted light, or by using a boron etch stop. This generic technology is used for the realization of different types of sensors and actuators or integrated compositions.

\section{Vibrating membrane systems}

The basic silicon membrane is achieved by anisotropic etching as shown in Fig. 2. The membrane can be brought into resonant vibration using one of several excitation methods. Inducing lateral stress in the membrane by means of an applied force, pressure or temperature elevation, the resulting shift of the resonance frequency is used as the sensor output.

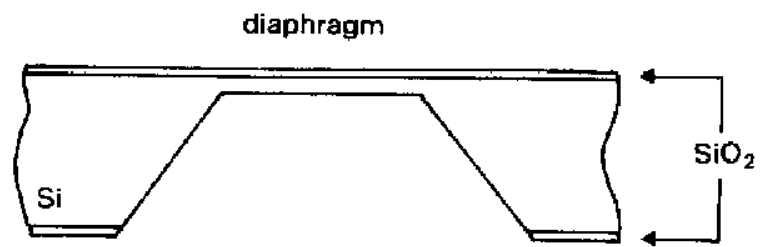

Fig. 2. Cross-section of anisotropically etched diaphragm in (100) silicon.

The piezoelectric-driven resonant diaphragm pressure sensor

The first developed resonant silicon diaphragm system makes use of a layer of $\mathrm{ZnO}$ with two pairs of gold bottom and top electrodes, deposited in the centre of the silicon diaphragm, as drawn in Fig. 3 [4]. Excitation of the diaphragm is accomplished by means of an a.c. voltage applied to one

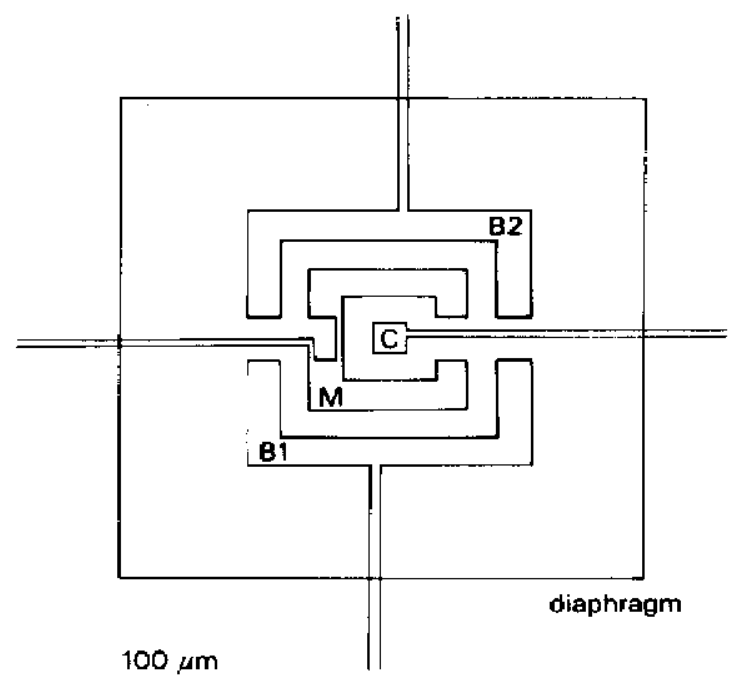

Fig. 3. Top view of diaphragm with bottom electrode, determining the active areas of the piezoelectric transducers (B1, M, C, B2). 
of the pairs of electrodes, the driver electrodes. Thereby, moments and forces are exerted by the piezoelectric $\mathrm{ZnO}$ on the diaphragm, bringing this into a transverse vibration. This vibration is detected by the second pair of electrodes, the receptor electrodes. The diaphragm with integrated driver and receptor can be considered as an electrical two-port network and be applied as such in the feedback loop of an amplifier. The oscillation frequency of this system will be determined by the resonance frequency of the diaphragm, and thus a frequency can be detected that is a function of the pressure applied to the membrane. The accuracy depends on the quality factor $Q$ of the system. Typical values of the mean resonance frequency are $200 \mathrm{kHz}$, a quality factor of 150 and a frequency shift of $0.1 \mathrm{~Hz} / \mathrm{Pa}$.

The thermally-excited resonant diaphragm pressure sensor

A similar system to that described above can also be developed with an electrothermal driver and a piezoresistive receptor, as shown in Fig. 4 [5]. In this case a d.c. and superimposed a.c. current through a diffused resistor in the centre of the diaphragm induces temperature changes over the silicon diaphragm, causing expansion and contraction in the silicon and resulting in a mechanical driving force. The receptor consists in this case of a diffused Wheatstone bridge at the edge of the diaphragm, giving a signal that is proportional to the periodic deformation. The system can again be considered, as in the previous case, as an electrical two-port network with a pressure-dependent transfer function. By putting this network in the feedback loop of an amplifier, the resulting oscillation frequency will also be pressure dependent. Typical values of the oscillation frequency are $120 \mathrm{kHz}$, a quality factor of 80 and a frequency shift of $0.25 \mathrm{~Hz} / \mathrm{Pa}$, which is a much higher sensitivity than that achieved with the piezoelectric driven type described above.

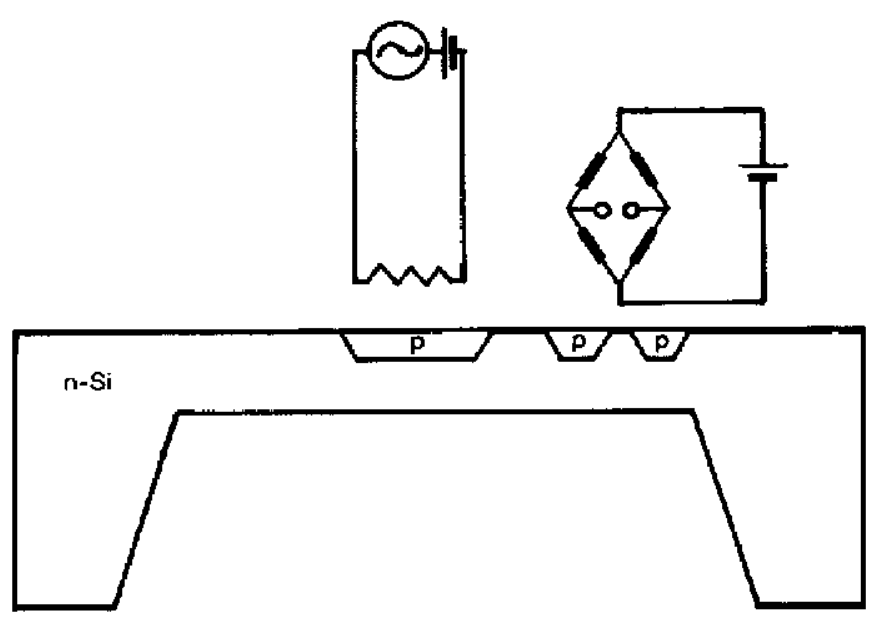

Fig. 4. Thermal excitation and piezoresistive detection. 
The thermally-excited resonating membrane mass-flow sensor

Another application of the thermally-excited resonating membrane is a mass-flow sensor [6]. In this case the system described above is placed in a gas flow. The heat generated in the central diaphragm resistor by a d.c. current is transferred to the gas and the resulting steady-state temperature of the membrane is dependent on the mass flow. Using again an excitationdetection feedback system, the resonance frequency will now be dependent on the mass flow. For a diaphragm with a thickness of $3 \mu \mathrm{m}$ and dimensions of $2 \times 2 \mathrm{~mm}$, a typical value of the frequency shift is $1 \mathrm{kHz}$ with a mass flow of $10 \mathrm{sccm}$.

Unfortunately heat is not only transferred to the gas, but is also lost to the chip substrate at the edges of the membrane. Therefore the newest design of this type of mass-flow sensor will consist of a bridge over an etched $V$-groove, which acts as the flow channel, while the bridge edges consist of suspended narrow beams. It is expected that the sensitivity of these sensors will increase drastically with respect to the original design. An SEM photograph of a V-groove with different suspended microstructures is given in Fig. 5.

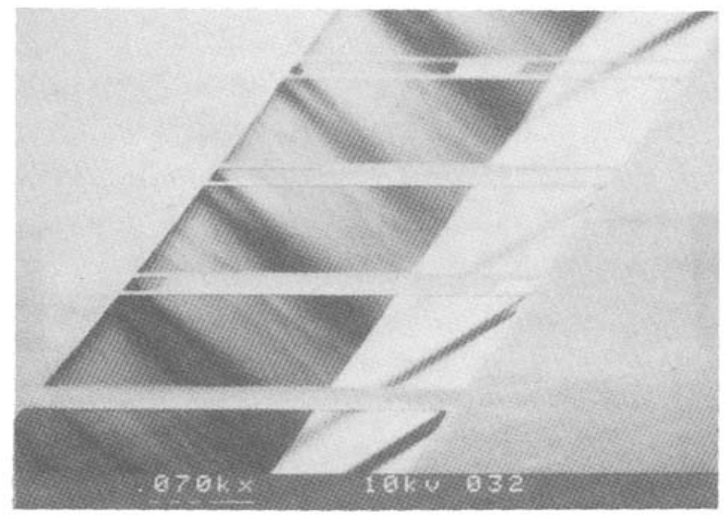

Fig. 5. SEM photograph of $V$-groove with suspended microstructures. Dimensions $V$ groove: width, $600 \mu \mathrm{m}$; depth, $210 \mu \mathrm{m}$. Dimensions microstructures: $600 \mu \mathrm{m} \times 100 \mu \mathrm{m} \times$ $3 \mu \mathrm{m}$, with suspension beams of $16 \mu \mathrm{m}$. (Note: these are bare structures.)

The combination of thermal insulation and small dimensions, made possible by micromachining, leads to very fast and highly-sensitive flow sensors with response times in the order of milliseconds, providing the user directly with a digital signal as a function of flow.

The optically-controlled resonating membrane pressure sensor

In another performance of the resonating silicon diaphragm, use is made of optically-induced thermal expansion, while an integrated optical displacement sensor detects the vibrations [5]. The diaphragm is now periodically heated by the absorbed light from an optical fibre. The resulting local temperature variations cause the silicon to expand and contract, which in fact is the mechanical driving force for the microstructure. The vibrations 


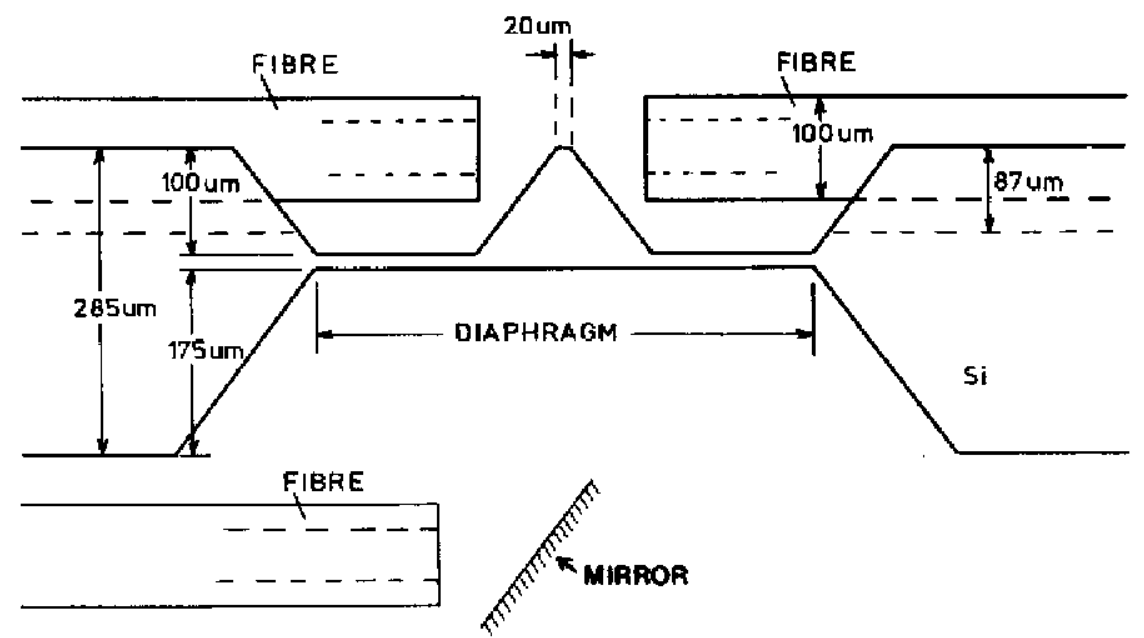

Fig. 6. Cross-section of optically-controlled resonating membrane pressure sensor.

are detected by an optical displacement sensor of the moving shutter type. Therefore a pyramid is part of the silicon diaphragm as the result of ingenious micromachining. Etched V-grooves on the top of the sensor chip are used for precision guiding of two optical fibres perpendicular to the silicon pyramid. A cross-sectional view of the whole system is given in Fig. 6 .

This integrated optical displacement sensor is connected to the input of a feedback amplifier, whose output controls the input optical driver. As in the other cases described above, the system will now oscillate at its resonance frequency, which is again a function of an applied pressure difference over the diaphragm. Typical values of the resonance frequency are $25 \mathrm{kHz}$ with a quality factor of 150 .

The resonating silicon beam force sensor

Another example of using a bridge as the heart of a sensor can be found in the force sensor [7], which is piezoelectrically driven by $\mathrm{ZnO}$ layers. The working principle is exactly the same as for the piezoelectrically-driven resonant diaphragm sensor, but in this case the pulling force on the bridge structure directly influences the resonance frequency. By choosing the proper dimensions, it is possible to make the frequency shift as large as several times the unloaded frequency, resulting in a sensor with a high resolution.

All the examples of resonating membranes and bridges described above show clearly how micromachining possibilities as well as the mechanical and thermal properties of silicon can be exploited with great advantage. Furthermore, all systems are examples of an integrated excitation-detection system in a feedback loop. Such systems have the advantage that if the quality factor of the closed-loop system is high, the requirements for the sensor itself, for instance the piezoresistive force sensor, are much less than in an open-loop application. 
Besides the projects described above, in which the micromachining is combined with integrated excitation and detection elements, other projects make explicit use of the micromachining facilities as such to construct devices in silicon. Examples are the micropump and the microphone cartridge.

\section{The piezoelectric-driven silicon micropump}

Various designs of micropumps have been developed, all based on glass/silicon/glass sandwich structures. The most sophisticated design is the three-valve pump, as shown in cross-sectional view in Fig. 7 [8].

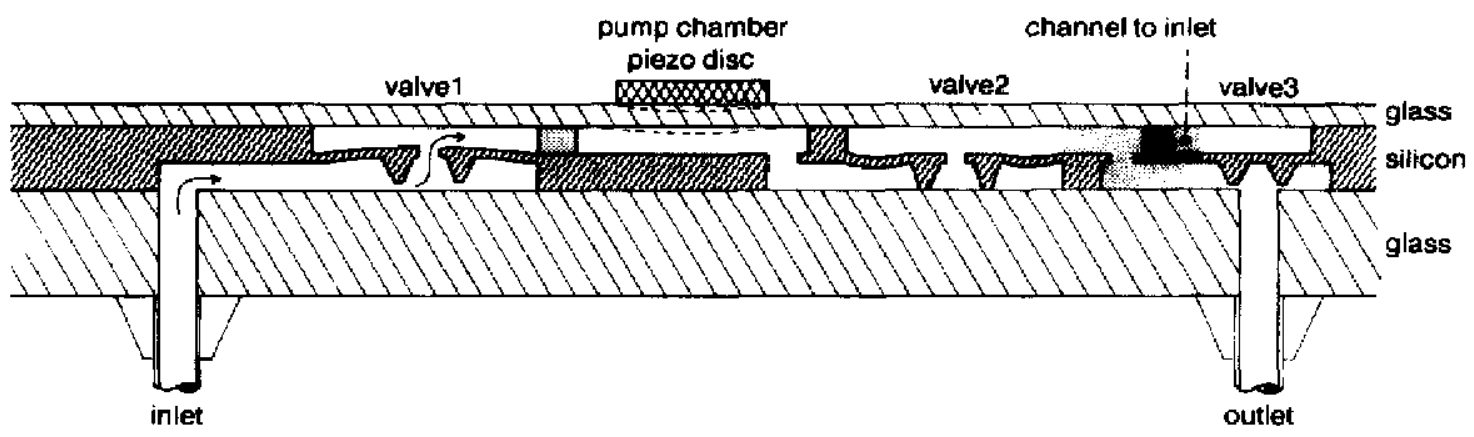

Fig. 7. Cross-section of piezoelectric-driven silicon micropump.

This pump consists of a pump chamber and three valves, all micromachined in one and the same silicon wafer, as well as the inlet, outlet and interconnection channels. This microstructure is sandwiched between two boron silicate glass plates, one acting as a support, having a thickness of $1.6 \mathrm{~mm}$, and one acting as the pump membrane, having a thickness of $0.3 \mathrm{~mm}$. The glass plates are sealed to the silicon by means of anodic bonding. Above the pump chamber, the glass membrane is provided with a Philips piezoelectric disk, glued onto the glass with Cyano acrylate adhesive. When this element is activated on one half cycle, fluid is drawn into the chamber, while in the next half cycle fluid is pumped out. The integrated silicon valves open and close automatically due to the pressure difference across them. With the output section, which is connected to the inlet by means of an internal etched channel, the influence of the output pressure on the total pumped volume is reduced to a minimum, which was not the case in the original two-valve pump. The pump driving is typically accomplished by $0.1 \mathrm{~Hz}$ pulsing at the piezo disk, resulting in a liquid flow of 0.5 microlitres/min, approximately independent of the outlet pressure up to $0.2 \mathrm{~atm}$. The micropump is aimed to be part of an implantable insulin delivery system.

\section{The microphone cartridge}

As in the previous example of micromachining, a chip microphone has been developed with silicon as the basic material [9]. By means of anisotropic etching a microphone backplate is constructed, including an air cavity, 


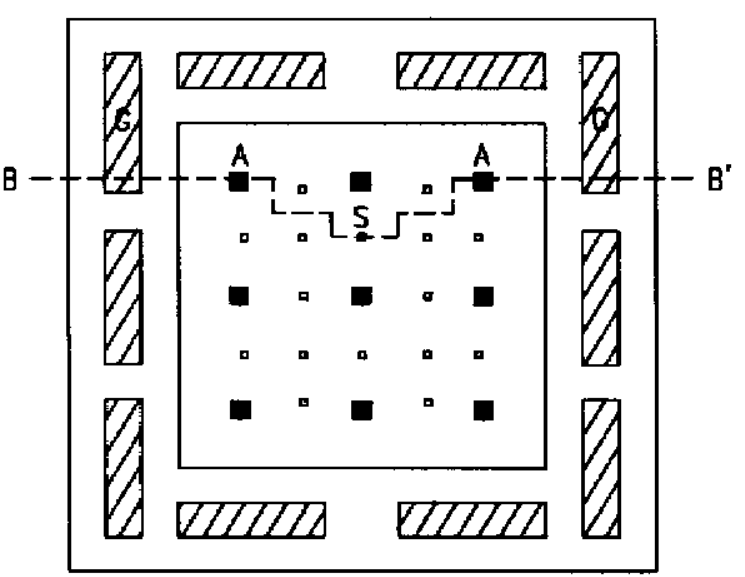

(a)

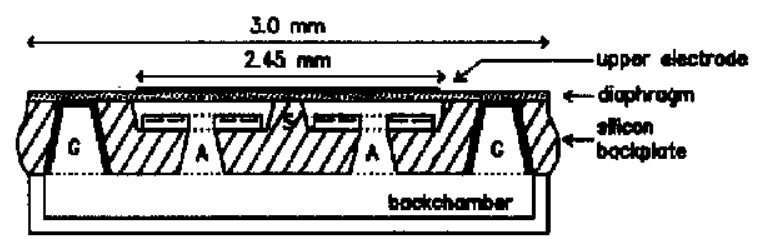

(b)

Fig. 8. Top view (a) and cross-section (b) along the line $B-B^{\prime}$, of the silicon electret microphone. For symbols see text.

membrane supports ( $\mathrm{S}$ in Fig. 8), a back chamber with air cavity holes (Fig. 8A) and membrane contact holes (Fig. 8G). This chip is covered with a Mylar membrane, which is partly covered with an evaporated electrode. The top view and the cross-section of the device are given in Fig. 8 .

The sensitivity of the capacitive pressure sensor can be greatly enhanced by incorporating an electret in the air cavity. Originally Teflon-FEP foils were deposited and patterned in the bottom of the air cavity, but at present the electret consists of chemically-treated silicon dioxide, which can be charged using a corona charging set-up, resulting in an equivalent voltage of $350 \mathrm{~V}$. The Mylar membranes are attached on complete wafers by means of spraying a polymer through the membrane contact holes (Fig. 8G). In this way 64 identical microphone cartridges can be manufactured in a 2 " wafer. The design is optimized for application in hearing aids and shows a sensitivity of $1.5 \mathrm{mV} / \mu \mathrm{bar}$ with a flat frequency response within $1 \mathrm{~dB}$ from $100 \mathrm{~Hz}$ to $15 \mathrm{kHz}$.

\section{Optical sensors}

For optical sensors use is often made of glass fibres, but the use of planar waveguides allows a far greater freedom of sensor design. Therefore, the optical sensor projects are focused on planar technologies. The devices are mainly based on the exploitation of mode-coupling principles. 


\section{Electric field sensor}

The mode-coupling principle can be exploited in a sensor for the measurement of electric fields at high-voltage power plants [10]. The sensor principle is illustrated with a device consisting basically of two parallel waveguides of $\mathrm{ZnO}$ and $\mathrm{Si}_{3} \mathrm{~N}_{4}$ provided with a periodic electrode array as schematically shown in Fig. 9.

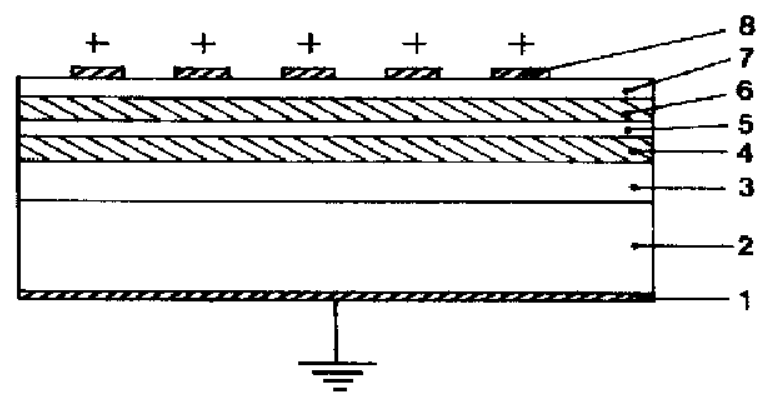

Fig. 9. Cross-section of electric field gensor. 1, aluminium ground electrode ( $1 \mu \mathrm{m}) ; 2$, silicon wafer $(280 \mu \mathrm{m}) ; 3, \mathrm{SiO}_{2}$ substrate $(1 \mu \mathrm{m}) ; 4, \mathrm{Si}_{3} \mathrm{~N}_{4}$ core $(260 \mathrm{~mm}) ; 5, \mathrm{SiO}_{2}$ buffer $200 \mathrm{~nm}) ; 6, \mathrm{ZnO}$ core $(300 \mathrm{~nm}) ; 7, \mathrm{SiO}_{2}$ buffer $(200 \mathrm{~nm}) ; 8$, aluminium top electrodes $(200 \mathrm{~nm})$.

An externally applied voltage will induce a periodic modulation of the refractive index of the electro-optic $\mathrm{ZnO}$ layer. This periodic perturbation forces light, propagating through the $\mathrm{ZnO}$ waveguide, to couple into the $\mathrm{Si}_{3} \mathrm{~N}_{4}$ guide, the coupling strength being dependent on the change in refractive index. Ultimately the value of the voltage can be deduced from the intensity ratio of the light output in the waveguides.

Opto-chemical sensors: the surface plasmon wqueguide (SPW) sensor

A surface-plasmon carrying structure is used as a modulator of the intensity of light propagating through a planar waveguide, consisting of a thin silver layer, covered with a very thin $(20 \mathrm{~nm})$ organic coating that contains particular chromoionophores. Figure 10 shows how the plasmon structure is deposited over a length of $100 \mu \mathrm{m}$ on top of the waveguide structure [11].

Polychromatic light is excited in the waveguide mode. Photons with equal propagation constants in waveguide- and plasmon-modes couple to the plasmon mode, where they are strongly damped. The wavelength at which this coupling is maximum depends on the optical constants of the organic coating, which are a function of the concentration of ions to which the chromoionophores are selectively sensitive. The sensor thus operates as an ion-selective absorption filter.

Silver is used as the plasmon-active material because of its low damping compared with other metals. Between this plasmon-sensitive layer and the chemically-sensitive layer, an intermediate layer of zirconia is deposited for matching the propagation constants of plasmon and waveguide modes as well as for maximizing the strength of the external part of the electric 


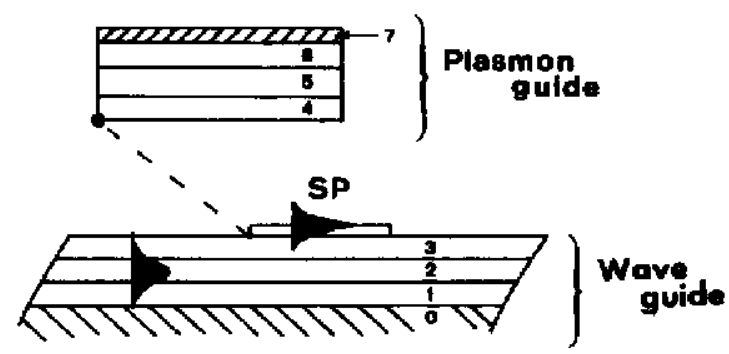

Fig. 10. Schematic cross-section of the SPW sensor.

$0=$ silicon wafer

$1=\mathrm{SiO}_{2}$ buffer (thermally oxidized), $d=0.5 \mu \mathrm{m}$

$2=$ SiON waveguide (LPCVD), $d=0.52 \mu \mathrm{m}$

$3=\mathrm{SiO}_{2}$ cladding (CVD), $d=0.4 \mu \mathrm{m}$

$4=\mathrm{TiO}_{2}$ (evaporated), $d=28 \mathrm{~nm}$

$5=A g$ (evaporated), $d=50 \mathrm{~nm}$

$6=\mathrm{ZrO}_{2}$ (evaporated) $d=512 \mathrm{~nm}$

7 = organic sensing layer, $1 \mathrm{~nm}<d<10 \mathrm{~nm}$.

field, which actually senses the organic layer. In this way the sensitivity for changes in the dielectric constant of the chemically-sensitive coating can be optimized. The surface plasmon mode is only excited at the silver-zirconia interface, because the intermediate layer of titania prevents excitation at the other silver interface. The underlying waveguide consists in this case of a silicon-oxygen-nitride ( $\mathrm{SiON}$ ) core, embedded between two silica layers and deposited on a silicon substrate. Preliminary experiments are very promising.

It will be obvious that the construction of this type of multilayered sensors puts severe demands on the technology. Smooth layers have to be deposited; only small tolerances in thickness and refractive index are allowed. For a proper operation of these opto-chemical sensors, special chromoionophores are being developed [11].

\section{Opto-chemical sensors: the luminescent quenching chemical sensor}

A planar waveguide optical sensor that is easier to construct is the luminescent quenching sensor [12]. In this case part of a planar waveguide has to be covered with an organic overlayer, consisting of a layer containing the luminescent centres and a monomolecular layer of potentially quenching molecules. These latter molecules are required to occur in two states: complexed with the chemical entity to be measured, they have to show quenching behaviour; uncomplexed, they must not quench at all. This type of sensor is schematically shown in Fig. 11. The waveguide now consists of a layer of silicon nitride embedded in silicon dioxide.

The sensing mechanism is based on the radiationless energy transfer between luminescent centres (donors) and quenching centres (acceptors) in the surface coating. The luminescense is excited by the monochromatic light in the waveguide mode, the intensity being modulated by the amount 


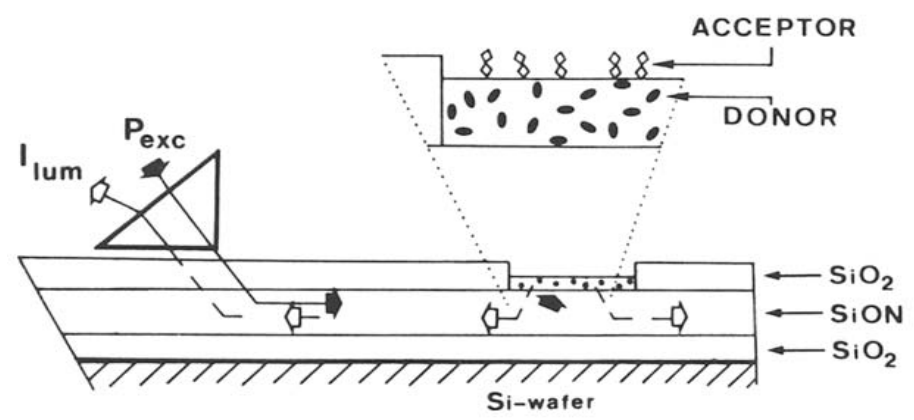

Fig. 11. Cross-section of the luminescent quenching chemical sensor.

of molecules that are in the quenching state, which is dependent on the concentration of chemical entities in the liquid surrounding the sensor.

In a first attempt to demonstrate the sensor operational mechanism, pH changes have been measured, using pH-indicator molecules (bromocresolpurper) as acceptors and dye molecules (rhodamine 6G) incorporated in a polyurethane layer as donors. The quenching rate, which is the sensor output, now appears to be a function of $\mathrm{pH}$. This sensor shows a large sensitivity, because even a small fraction of the centres in the monomolecular layer, being in the quenching state, can be detected easily.

Immunosensor based on surface plasmon resonance

An essentially even simpler but more bulky construction of a surface plasmon sensor is based on the attenuated total reflection of a silver-coated glass slide [13]. By measuring the reflectance of monochromatic light on the metal-coated glass surface as a function of the angle of incidence, plasmon resonance can be detected as a sharp minimum in the reflectance at a certain angle. Optical properties like the effective refractive index of a sensing layer positioned within the evanescent field of the surface plasmon will modulate this specific angle. Figure 12 shows a set-up by which this angle can be measured.

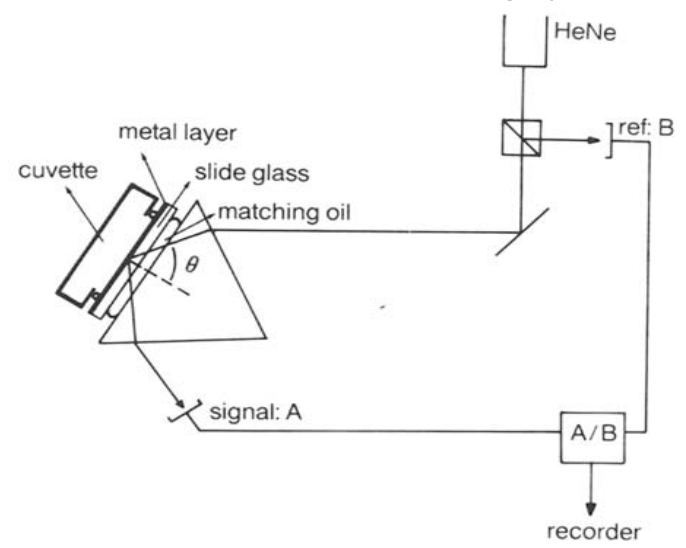

Fig. 12. Experimental set-up for the detection of surface plasmons. The reflectance is recorded as a function of $\theta$. 
If the cuvette contains proteins that adsorb at the metal layer, the adsorption process can be monitored by recording the reflectance as a function of time for a fixed angle of incidence. If, furthermore, the adsorbed proteins are antibodies (antigens), an immunoreaction that changes the effective refractive index of the adsorbed layer can be monitored as soon as the corresponding antigens (antibodies) are added to the cuvette. A concentration of approximately $10^{-10} \mathrm{~mol} / \mathrm{l}$ antibody could be detected with an aHSA/HSA experiment.

\section{Recording media and sensors based on magnetic materials}

Fundamental research on magnetic materials has already been carried out for many years in the Faculty of Electrical Engineering. Two different materials are principally of interest with respect to the S\&A group, namely $\mathrm{CoCr}$ and its derivatives and $\mathrm{NiFe}$. $\mathrm{CoCr}$ is a hard magnetic material especially useful for the storage of information, while NiFe is a soft magnetic material, applicable for magnetic sensors. Examples will be given below concerning material research projects as well as applications in the field of magnetic recording and various magnetic sensors.

\section{CoCr research}

In order to establish stable information storage in CoCr films, it is of great importance to understand in detail the mechanisms of magnetization on a microscale, e.g., domain formation and the magnetic reversal process. If the reversal mechanism takes place by rotation of the magnetic moment of a distinguishable microcolumn of $\mathrm{CoCr}$, then the column boundaries coincide with the domain boundaries. However, should the reversal mechanism be the result of domain wall movement, then the columns are magnetically coupled. The micromagnetic behaviour of a CoCr film is strongly related to the mechanism of the reversal effect, which can be controlled by the preparation method [14]. Usually CoCr films are r.f. sputtered on hard substrates such as silicon, glass or aluminium and soft substrates such as polyimide and PET. Various parameters of the sputtering process as well as the type of substrate appear to determine the nucleation and growth of the layer. For optimizing the read process, a soft magnetic NiFe layer is used between the substrate and the CoCr layer. To stimulate the columnar morphology and the texture of the CoCr layer, seed-layers of Ti, Si etc. are also used. Figure 13 is an SEM picture of a $1 \mu \mathrm{m}$ thick sputtered CoCr layer on a Si substrate, showing the columnar morphology of such a film.

The aim of the CoCr research project is to manufacture magnetic films especially suitable for information storage with very high bit densities, applicable in perpendicular recording. In the field of high-density recording, several other materials are being investigated, such as $\mathrm{CoFeO}$, made by 

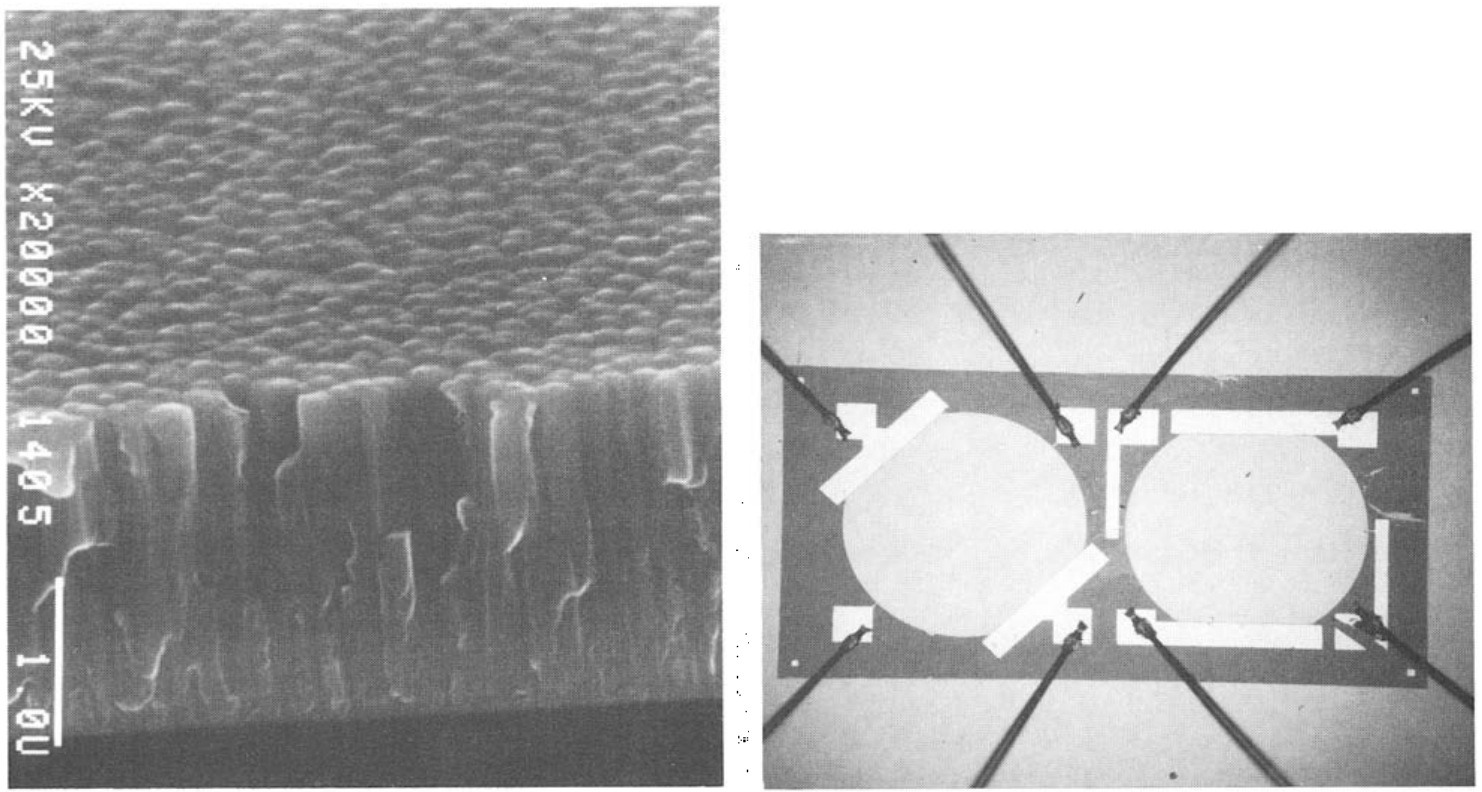

Fig. 13. Cross-gectional SEM structure of a sputtered CoCr film with columnar morphology.

Fig. 14. Photograph of two Hall elements, placed close together at an angle of $45^{\circ}$.

reactive evaporation, and other Co-like materials, made by the angle of incidence deposition process.

\section{NiFe research}

Contrary to CoCr described above, NiFe (Permalloy) is a rather soft ferromagnetic material, which makes it very suitable for the measurement of small magnetic fields and thus for a class of sensors based on modulation of magnetic fields. Permalloy layers are obtained by evaporation or by r.f. sputtering on various types of substrates, depending on the particular type of sensor to be constructed. Research on NiFe films is concentrated on optimization of the physical properties, such as magnetic and resistance anisotropy, in relation with the photolithographic determination of specific geometries, which are necessary for the construction of devices for contactless angle detection, magnetic field measurements and absolute position detection.

Sensor for contactless angle detection

The contactless, and thus friction free, measurement of an angle can be realized by the use of a planar Hall element, which senses the position of a permanent magnet. The Hall element consists of a Permalloy film, deposited on a certain substrate. The output voltage of a single Hall element will, however, be non-linear and temperature dependent, resulting in serious measurement errors. Improvement of the accuracy of such a sensor can be achieved by using two of these elements placed close together at $45^{\circ}$ to each other. The design is shown in Fig. 14 [15]. The circular Permalloy layers, with a diameter of $900 \mu \mathrm{m}$ each, are deposited on an oxidized silicon wafer 
with aluminium contacts. In this way an accuracy of angle detection is achieved of the order of $0.05^{\circ}$. In the near future the necessary electronic circuitry will be integrated with this angle detector.

\section{Thin-film magnetometer}

A simple planar Permalloy film can also be used to measure magnetic fields, which is in fact the original application of planar Hall elements. Uncontrolled formation of magnetic domains may, however, decrease the sensitivity of the Hall voltage with respect to the magnetic field to be measured. On the other hand, this negative effect can also be exploited by modulating the domain formation in a controlled way. Therefore, an alternating driving field must be applied, oriented in the plane of the Permalloy film and perpendicular to its easy axis of magnetization. The amplitude of this excitation field should be such that the film will periodically be saturated in a positive and in a negative direction. This $50-50 \%$ rotation results in a zero Hall voltage, but the ratio will be disturbed by any easy-axis field (the measurand), resulting in pulsating Hall voltages. By relatively simple electronic processing, this a.c. Hall voltage can be converted into a voltage proportional to the magnetic field to be measured. With given dimensions of the Permalloy film, the complete magnetometer has been miniaturized by integration of the driving field coil. An open view is given in Fig. 15 [16].

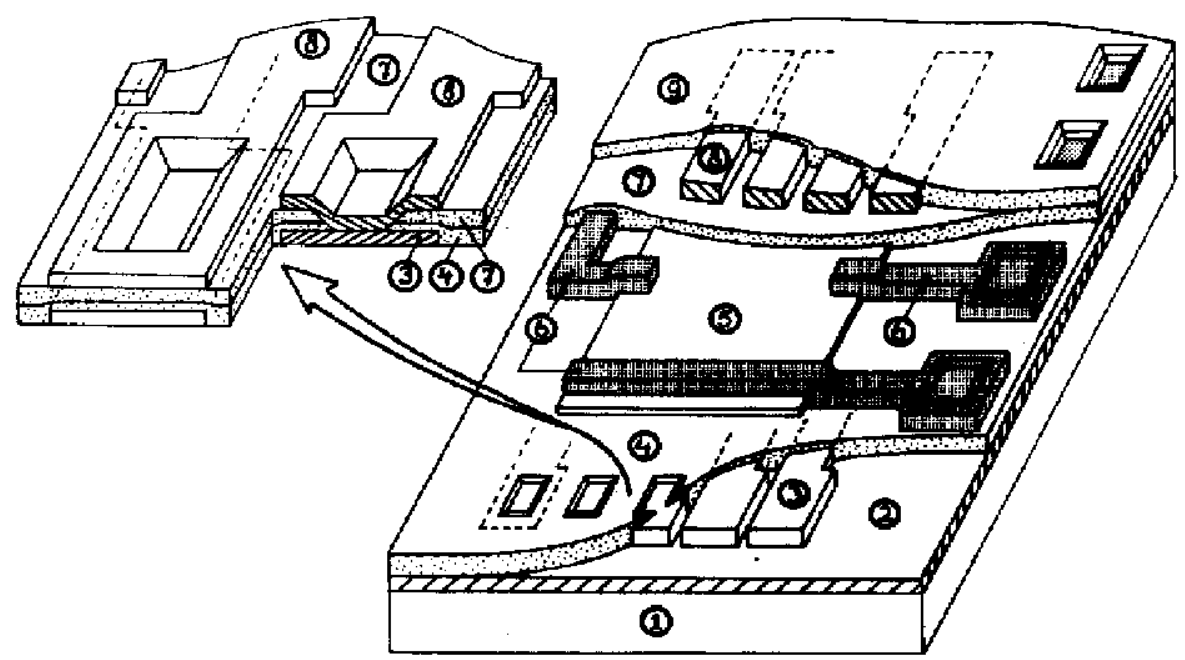

Fig. 15. Open view of thin-film magnetometer construction including a planar drive coil.

$1=\mathrm{Si}$ substrate

$\mathbf{2}=\mathrm{SiO}_{2}$ insulator

$3=$ Al lower half of coil

$4=$ polyimide insulator

$\mathbf{5}=$ permalloy

$6=$ Al contacts to permalloy

7 = polyimide insulator

$8=$ Al upper half of coil

$9=$ polyimide insulator. 
The NiFe film is deposited on an oxidized silicon wafer, which was first provided with aluminium strips, covered with polyimide, thus forming the lower part of the coil. On top of these layers, polyimide is again used as an insulating layer, which is covered with aluminium strips, interconnected with the lower strips, thus forming the complete coil. Aluminium is also used to connect the Hall element. Preliminary results show a resolution of $10^{-8}$ Tesla, which means that the earth's magnetic field can be measured rather well, making it possible to realize in principle a very small compass with electrical output.

\section{Absolute position sensor}

If a hard magnetic layer contains a digital bit pattern, the movement of this layer can be detected by a Permalloy strip (array), which is positioned with its plane perpendicular to the hard magnetic layer and parallel to its direction of movement. Because the entire bit combination that represents the position coordinate can be detected in a static situation, the sensor principle is absolute in nature. Although it appears to be difficult to position the Permalloy strips just at the edge of the (silicon) substrate, prototype sensors show that it is possible to measure position over a range of 1 meter with a resolution of $50 \mu \mathrm{m}$ [17]. To investigate the effect of the strip geometry, the prototype sensor head is divided into different separate sensor elements by means of the aluminium contact pattern. This can be seen in Fig. 16.

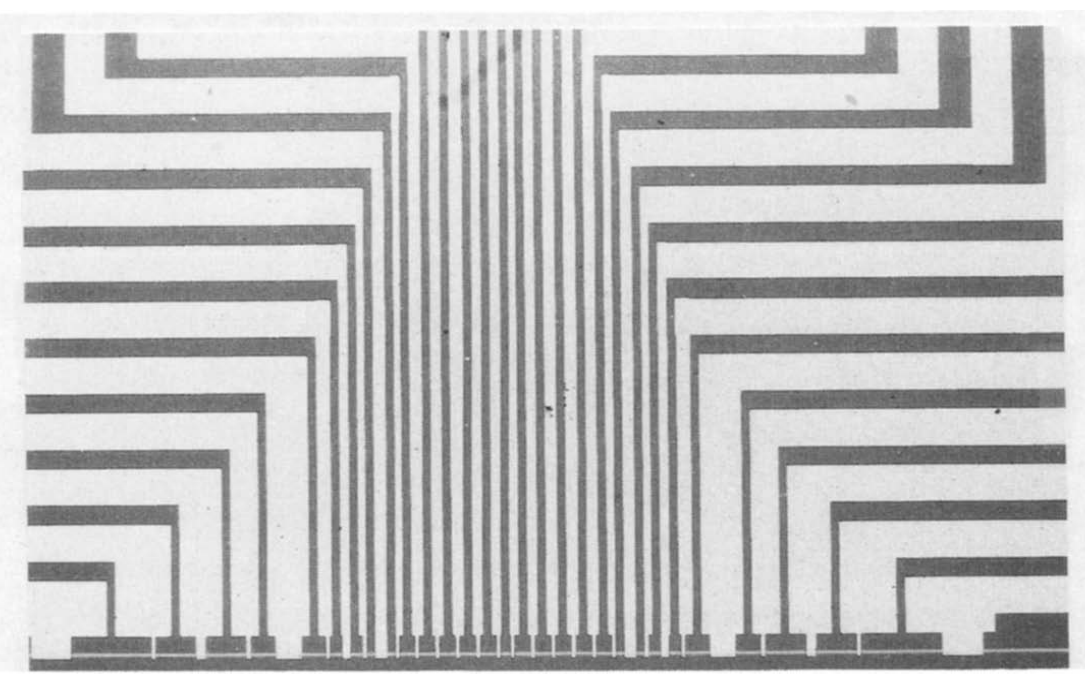

Fig. 16. Layout of experimental position-detection head. The permalloy sensor strip is divided into separate sensor elements by means of the aluminium lead pattern.

\section{FET-based sensors}

As may be known, the first papers on ISFETs originate from the University of Twente $[1,2]$. Since then, the research efforts have concentrated in the first place on clarifying the operational mechanism of 
devices of this type having inorganic gate materials. It appeared that the operation could be described by applying the site-dissociation model to the electrolyte/gate interface, resulting in the definition of a specific sensitivity parameter, characteristic for each type of inorganic gate material [18].

With respect to the application, the research and development activities were focused on the construction of a pH catheter-tip sensor, in cooperation with Cordis, later on Sentron, both established in Roden, the Netherlands. This resulted in the commercialization of a clinical device, proven to be useful during surgical operations for monitoring the effect of mechanical ventilation, especially at extubation. The inherent drift of ISFETs was solved by Sentron by incorporating a PROM in the connector of the catheter, containing all essential device parameters, including the predetermined drift in $\mathrm{mV} / \mathrm{h}$ [19] of the particular ISFET chip mounted in the catheter-tip.

At the University research was further focused on the projects described below.

\section{Tantalum pentoxide ISFETs}

$\mathrm{Ta}_{2} \mathrm{O}_{5}$ appears to be a serious candidate for application as an inorganic gate material. For reasons of simplicity, we have chosen the evaporation or sputtering of tantalum on silicon dioxide, followed by photolithographic patterning and thermal oxidation [20]. Sensitivity and stability are, however, a function of the preparation process. Experimental results show that the highest sensitivity combined with the lowest drift rate can be achieved by using:

(i) a low substrate temperature during evaporation or sputtering;

(ii) a high-purity Ta source;

(iii) a low pressure during evaporation and sputtering;

(iv) thermal oxidation at $500^{\circ} \mathrm{C}$ in extremely dry oxygen atmosphere;

(v) a layer of tantalum no thicker than $50 \mathrm{~nm}$.

Obeying these process rules, a reproducible sensitivity of $58 \mathrm{mV} / \mathrm{pH}$ could be achieved, combined with a drift of $0.1 \mathrm{mV} /$ hour.

\section{Polymeric gate ISFETs (REFETs and CHEMFETs)}

The first task in developing polymeric-gate ISFETs is the reduction of $\mathrm{pH}$ sensitivity of the silicon dioxide gate by chemical modification. Subsequently the modified surface should be covered with specific crown ethers, all or none of which are incorporated in a thin polymeric insulating membrane. The first item could not be achieved, because any treatment with silylating agents was unable to reduce the number of surface sites to the $0.01 \%$ required for complete $\mathrm{pH}$-insensitivity, as calculated with the site-dissociation theory. Covering the remaining sites with a very thin perfectly impermeable and insulating polymeric layer also appears to be impossible, because pinhole-free layers could not be produced. Thicker pinholefree layers can be made, however, but these decrease the electrical sensitivity and thus the stability of the FET device too drastically. To solve this problem, ion-conducting polymeric layers, such as acrylates, have been used with 
success. To show no permselectivity, these layers are trimmed by the addition of immobile lipophilic cations, such as di-dodecyldimethylammonium bromide (DDMAB) [21].

Without the addition of ionophores, the device mentioned above can be used as a REFET, because of its insensitivity to any particular type of ion and independence of electrolyte concentration. Such a device has the same electrical sensitivity as the original underlying ISFET due to the conducting nature of the polymeric layer. In this way excellent differential ISFET/REFET pairs have been produced, which can measure ion activity with a noble metal pseudo-reference electrode for grounding the analyte.

On adding specific ionophores to the polymeric layer, selectivity for one particular ion can be achieved. As an example, potassium-sensitive devices have been produced using dibenzo-24-crown-8 incorporated in a PVC membrane.

In the REFET as well as the potassium-sensitive CHEMFET, the stability had to be improved, because the polymer/SiO ${ }_{2}$ interface still contains unreacted hydroxyl groups and is therefore still $\mathrm{pH}$ sensitive. Because the membranes contain water through which gases will diffuse, the devices can thus be influenced by the partial pressure of $\mathrm{CO}_{2}$. Therefore, an intermediate layer of buffered poly-HEMA has been introduced between the polymer and the gate of the ISFET. So the final design of the polymeric gate ISFETs is as shown in Fig. 17.

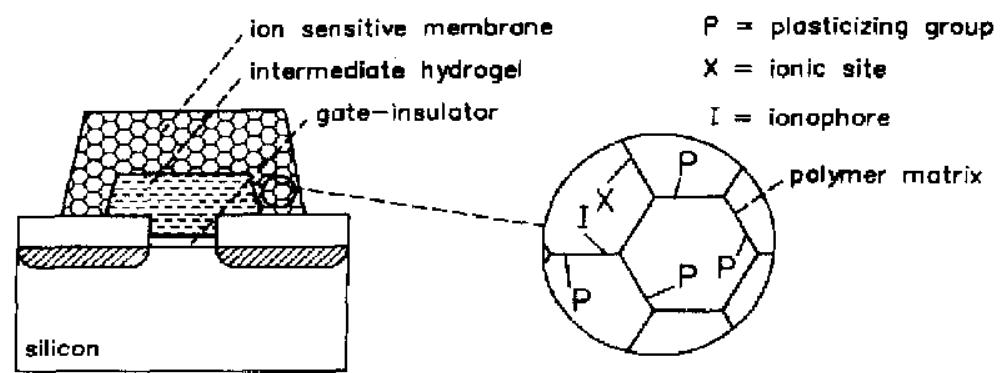

Fig. 17. Schematic representation of CHEMFET device.

To increase the lifetime of the devices, all layers are attached to each other by chemical bonds. Furthermore, the layers are deposited by spinning techniques and patterned by means of photolithography, for which purpose the mask aligner has been adapted to take control of the environmental condition during polymerization of the layers.

At present, CHEMFET devices are being developed for the measurement of heavy metal ions, while in the future ligands will also be synthesized for the measurement of anions.

\section{ISFETs with Coulometric facilities}

Conventionally, chemical sensors convert information from the chemical domain into the electrical domain unidirectionally. In practice, sensors have to be recalibrated at regular intervals, the length of which depends on 
the required accuracy in relation to the expected baseline drift rate and sensitivity changes. In this respect ISFETs do not differ from other ion sensors. However, due to their planar construction, small dimensions and fast response, ISFETs offer unique possibilities of being integrated with a $\mathrm{pH}$ actuator closely around the gate area. For instance, a gold electrode can easily be deposited with vacuum evaporation and be applied as a Coulometric electrode with respect to a remote counter electrode. In this way hydrogen or hydroxyl ions can be generated in the direct vicinity of the gate. Because the ISFET is now only used as an indicator electrode over a very short time, the 'Coulometric sensor' is not subject to changes in offset and sensitivity of the ISFET. In fact the information in the output signal of the system no longer consists of the absolute value of a voltage, as is usual, but of the time interval between the onset of the Coulometric pulse and the deflection time of the measured titration curve. In other words, static ISFET measurements are replaced by dynamic ISFET measurements with their corresponding advantages [22].

With a Coulometric sensor as described above, tiration curves have been recorded within seconds with closed and open systems, from which the concentration of acids and bases could easily be calculated.

A smart application of the Coulometric sensor is the ISFET-based Severinghaus electrode, in which the inner electrolyte is Coulometrically titrated by means of an integrated pair of electrodes [22] instead of using the ISFET directly as a pH electrode as would conventionally be the case. A cross-sectional view of the construction of this type of sensor-actuator system is shown in Fig. 18.

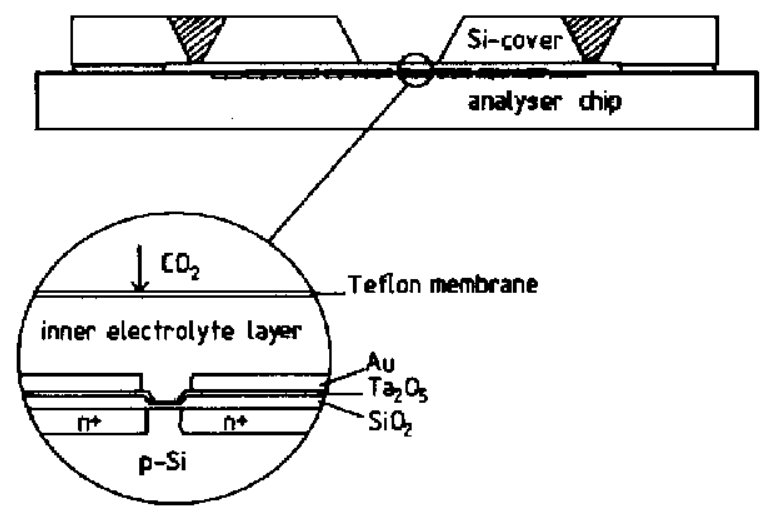

Fig. 18. Cross-section of Coulometric carbon dioxide sensor.

Carbon dioxide diffuses through the Teflon membrane, thus determining the $\mathrm{pH}$ of the internal electrolyte. The resulting acidity is measured by means of a back titration, independent of the ISFET offset and sensitivity. The long-term stability of the system is some orders of magnitude better than that of a classical Severinghaus electrode. 
ISFET-based pH-static enzyme sensor

ISFETs, covered with an organic layer containing a specific immobilized enzyme, can be used to measure the concentration of the corresponding substrate if the specific enzymatic reaction produces hydrogen or hydroxyl ions. These devices suffer, however, from non-linearity and buffer dependency, which makes the application hardly worthwile. However, if the layer with immobilized enzymes contains a Coulometric electrode (see above), the $\mathrm{pH}$ inside the membrane can be kept constant by means of a feedback loop [23]. This keeps the enzyme activity, which is a function of the $\mathrm{pH}$, constant and optimal, thus preventing the saturation effects that cause the non-linearity mentioned above. Furthermore, the system operates independent of the buffer capacity, because the 'enzymatic pH actuator' as well as the Coulometric $\mathrm{pH}$ actuator are both positioned in one and the same layer and thus depend equally on the internal buffer capacity.

Experimental results of a urea sensor without and with Coulometric feedback are shown in Fig. 19. Note that in the case of an open-loop ureasemodified ISFET, the measurand is the output voltage of the ISFET amplifier system, while in the case of the closed-loop (pH-static) system, it is the controlled current through the integrated Coulometric electrode.
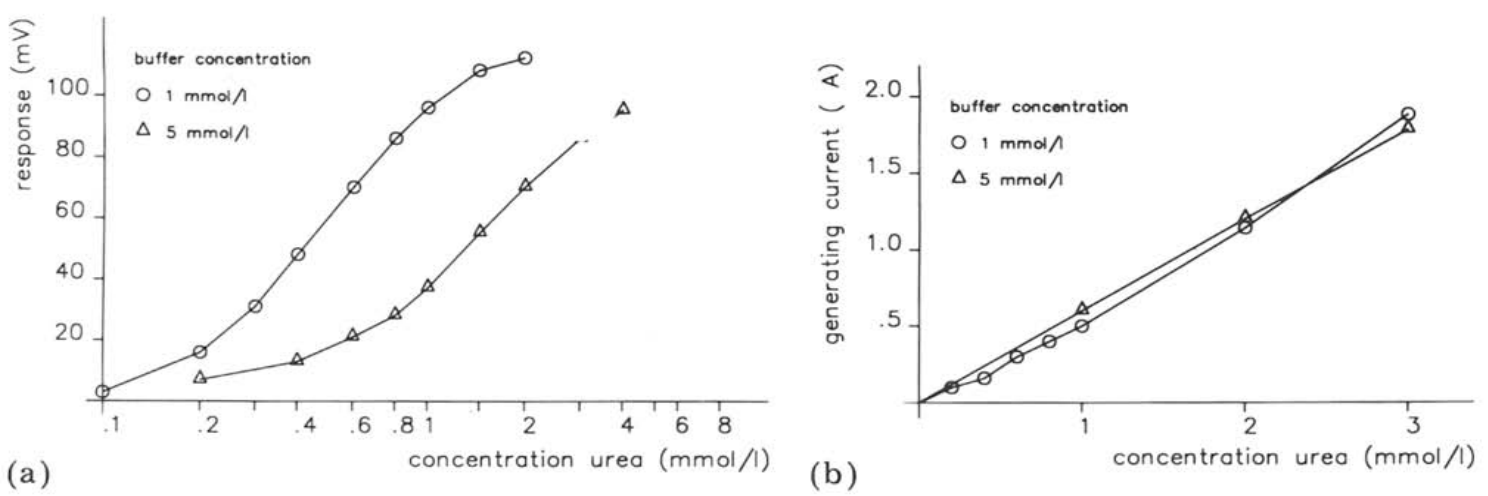

Fig. 19. (a) Output of urea ENFET without feedback. (b) Output of urea ENFET with feedback. Buffer concentration: $(O): 1 \mathrm{mmol} / \mathrm{l} ;(\Delta): 5 \mathrm{mmol} / \mathrm{l}$.

\section{Immuno-modified ISFETs}

It has long been discussed in the literature whether it would be possible to determine the specific protein charge of an antibody/antigen complex, covering an ISFET, and distinguish it from the charge of one of the separate constituents. This appears to be impossible using the immuno-modified FET (IMFET) in a static way, as is usually the case with ISFETs. This is due to the porous structure of the membranes, including the proteins, which are moreover surrounded by neutralizing counterions, thus shielding any electric dipole that may exist and be modulated by a specific immunoreaction.

The existence of a porous membrane, containing fixed immobile charges and mobile counter charges, can, however, be exploited using $\mathrm{dy}$ - 


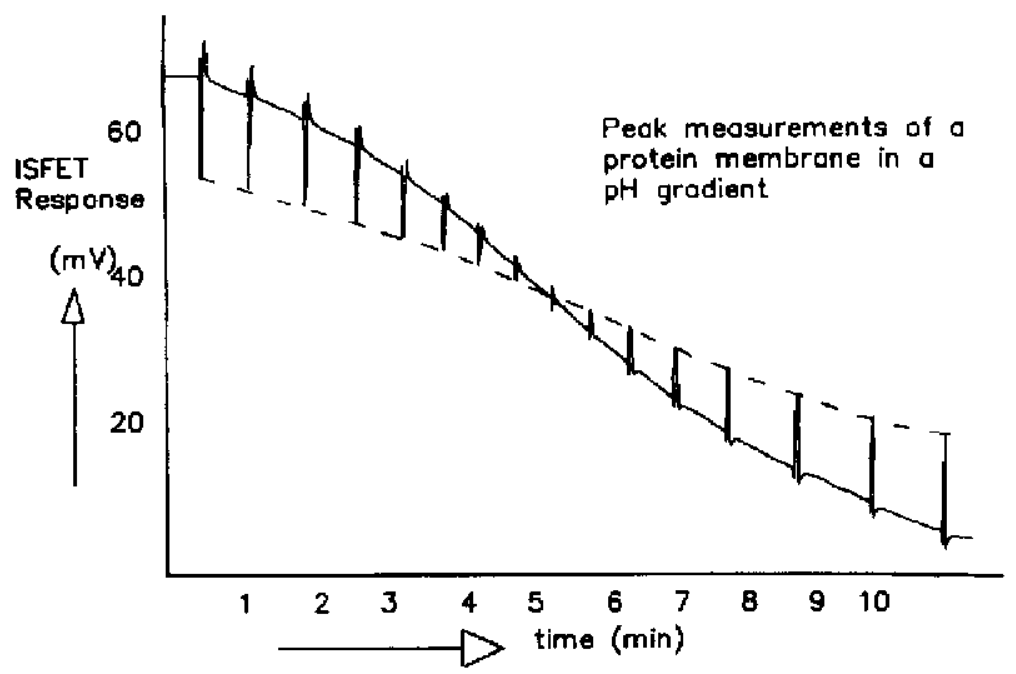

Fig. 20. Response of protein-modified ISFET on expsoure to $\mathrm{pH}$ gradient with additional repeated increases of electrolyte concentration.

namic measurements [24]. If, at a certain $\mathbf{p H}$, differing from the isoelectric point of the protein (complex) that is immobilized in a membrane covering the ISFET, a sudden change in the ionic concentration of the electrolyte is created, a temporary disturbance of the membrane potential will occur. This is the result of the effective mobility differences of cations and anions, determined by the value of the fixed charge density in the membrane. In the ISFET registration this means that the static output voltage of the ISFET amplifier, determined by the $\mathrm{pH}$ of the electrolyte, exhibits a pulseshaped disturbance, with an amplitude that is related to the fixed charge density in the membrane. Because this charge density is a function of the $\mathrm{pH}$ of the electrolyte, the isoelectric point of proteins can also be determined by gradually changing the electrolyte $\mathrm{pH}$, as shown in Fig. 20.

In this way also the difference in isoelectric points of an antibody (or antigen) immobilized in the ISFET membrane and the antibody/antigen complex after an immunoreaction can be determined. A membrane consisting of a polystyrene matrix with immobilized HSA clearly shows a point of zero fixed charge at a $\mathrm{pH}$ of 4 , while after the immunoreaction with aHSA, this typical point shifts to a $\mathrm{pH}$ value of 4.6. In the future the measuring procedure will be simplified, but it will be clear at this point that dynamic measurements with changing buffer concentration are a unique method of distinguishing between the characteristic isoelectric points of proteins, including antibodies, antigens and antibody/antigen complexes.

\section{Integration of sensor electronics}

In nearly all sensor projects described in the previous Sections, the main attention has been paid to the exploration of the different sensor operational mechanisms in relation to the appropriate technologies. Some of the projects have now progressed so far that the integration of specific 
electronic circuitry is also taken in hand. In the S\&A laboratory use can be made of an NMOS process line, while in collaboration with the Integrated Technology and Electronics Group, the $2.5 \mu \mathrm{m}$ twin-well CMOS process line can also be exploited. Up to now this CMOS process has been used with success for the development of a temperature sensor array with only two interconnecting leads.

\section{Temperature sensor array}

For the measurement of tissue temperature profiles during hyperthermic treatment of cancer tissue, catheters are necessary that contain a large number of temperature sensors at intermediate distances of about $0.5 \mathrm{~cm}$. In order to prevent lateral temperature leakage along the catheter, the sensors have to be interconnected with a minimum of wires, if possible only two. This implies that the sensor chips should also contain a certain amount of smart electronic circuitry for multiplexing and sensor signal modulation [25].

Therefore a multiplex principle has been developed by which each sensor chip is connected sequentially to two interconnecting leads, which carry, besides the supply voltage, also the switching pulses to control the multiplex circuit and the frequency-modulated (temperature) sensor signal from the sensor chip that is connected at a particular moment. Thus, each identical (all CMOS) chip contains a multiplex circuit, a temperature-tocurrent converter and a current-controlled oscillator. Figure 21 shows one sensor chip of $0.5-1 \mathrm{~mm}$, from which 50 can be connected at distances of $0.5 \mathrm{~cm}$ to two interconnection leads and sequentially be activated and read out.

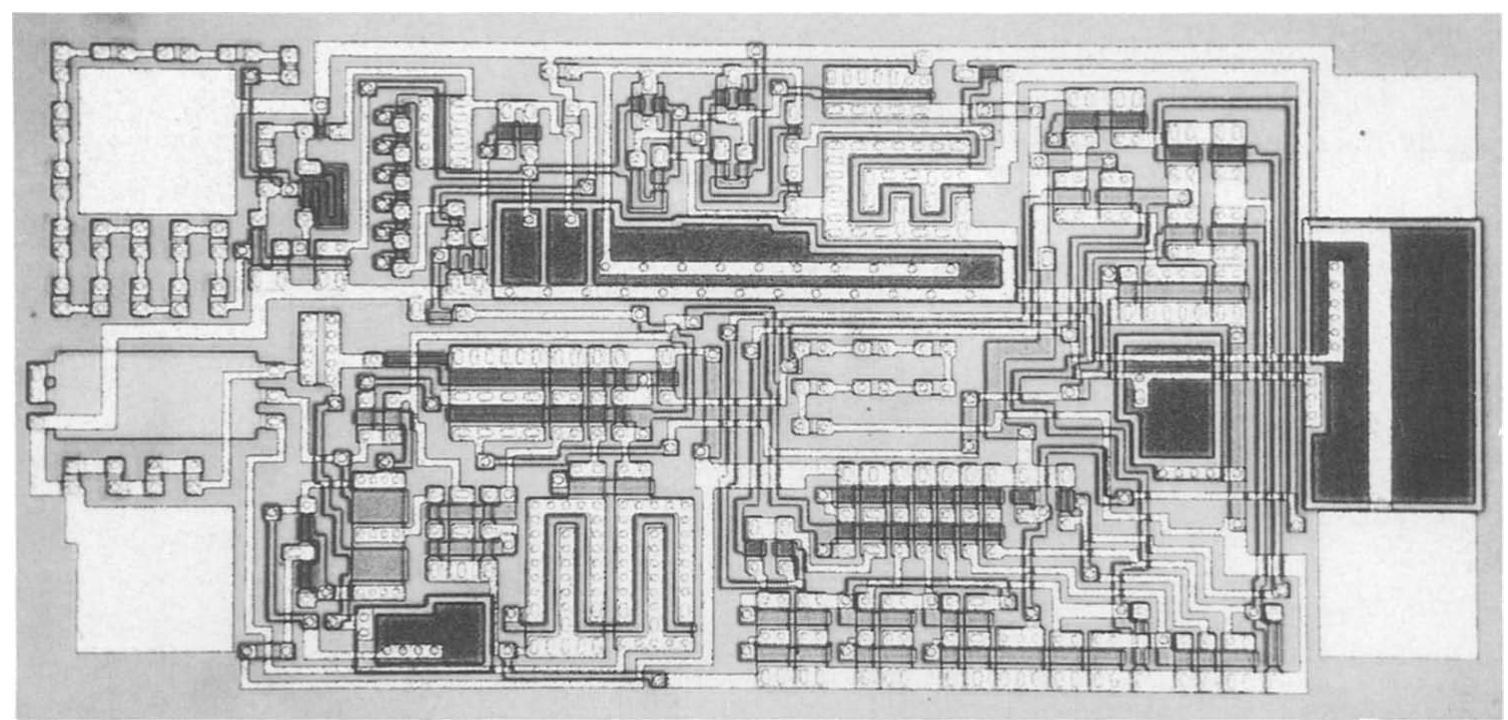

Fig. 21. Photograph of temperature sensor chip with integrated modulator and multiplex circuitry. Dimensions: $0.5 \times 1 \mathrm{~mm}^{2}$. 


\section{Acknowledgements}

This paper could only have been written with the help of the S\&A project leaders and Ph.D. Students actively involved with the different projects. Many of these projects are included in the programme of the Foundation for Fundamental Research on Matter (FOM), or are supported by the Netherlands Technology Foundation (STW). Some of the projects are part of an industrial contract.

\section{References}

1 P. Bergveld, Development of an ion-sensitive solid-state device for neurophysiological measurements, IEEE Trans, Biomed, Eng., BME-17 (1970) $70-71$.

2 P. Bergveld, Development, operation and application of the ion-sensitive field effect transistor as a tool for electrophysiology, IEEE Trans. Biomed. Eng., $B M E-19$ (1972) $342-351$.

3 J. H. J. Fluitman and H. W. Krabbe, Miniature sensor for two-dimensional magnetic field distributions, J. Phys. E: Sci. Instrum., 5 (1972) 963 - 964.

4 J. G. Smits, H. A. C. Tilmans and T. S. J. Lammerink, Pressure dependence of resonant diaphragm pressure sensor, Proc. 3rd Int. Conf. Solid-State Sensors and Actuators, (Transducers '85), Philadelphia, PA, U.S.A., June 11-14, 1985, pp. 93 - 96.

5 T. S. J. Lammerink and S. J. Gerritsen, Fiber-optic sensors based on resonating mechanical structures, Proc. Fiber Optic Sensors II, SPIE, 798 (1987) 67 - 71.

6 S. Bouwstra, P. Kemna and R. Legtenberg, Thermally excited resonating membrane mass flow sensors, Proc. Eurosensors I, Cambridge, U.K., 1987, pp. 109 - 113.

7 F. R. Blom, S. Bouwstra, J. H. J. Fluitman and M. Elsenspoek, Resonating silicon beam force sensor, Senzors and Actuators, 17 (1989) 513.

8 H. T. G. van Lintel, F. C. M. van de Pol and S. Bouwstra, A piezoelectric micropump based on micromachining of silicon, Sensors and Actuators, 15 (1988) $153-167$.

9 A. J. Sprenkels, R. A. Groothengel, A. J. Verloop and P. Bergveld, Development of an electret microphone in silicon, Sensors and Actuators, 17 (1989) 509.

10 W. H. G. Horsthuis and R. Pannekoek, Electro optic modulators in multilayered zinc-oxide waveguides, Springer Series in Optical Sciences, Integrated Optics, Vol. 48,1985 , pp. $152-157$.

11 J. van Gent, H. J. M. Kreuwel, P. V. Lambeck, J. Gerritsma, E. J. R. Sudhölter, D. N. Reinhoudt and Th. J. A. Popma, Opto-chemical sensor based on chromoionophores, Sensors and Actuators, 17 (1989) 297 - 305.

12 H. J. M. Kreuwel, P. V. Lambeck, J. van Gent and Th. J. A. Popma, Surface plasmon dispersion and luminescence quenching applied to planar waveguide sensors for the measurement of chemical concentrations, Fiber Optic Sensors II, SPIE, 798 (1987) $218-224$.

13 R. P. H. Kooyman, H. Kolkman, J. van Gent and J. Greve, Surface plasmon resonance immunosensors: sensitivity considerations, Anal. Chim. Acta, 213 (1989) 35 - 45.

$14 \mathrm{~J}$. C. Lodder and Li Cheng-Zhang, The influence of the segregated micro-structure on the magnetization reversal in CoCr films, J. Magn. Magnetic Mater, 74 (1988) $74-86$.

15 K. J. Eykel and J. H. J. Fluitman, Angle detection based on the resistance anisotropy of permalloy, IEEE Trans. Magn., MAG-22 (1986) 955 - 957.

16 R. Groothengel and R. M. de Ridder, Thin film magnetometer with integrated planar drive coil, in J. C. Lodder (ed.), Proc. 3rd Sensors and Actuators Symposium, Kluwer Techn. Books, Deventer, The Netherlands, 1986, pp. $211-218$.

17 J. P. J. Groenland, Magnetoreistive transducer for absolute position, IEEE Trans, Magn., MAG-20 (1984) 969 - 971. 
18 L. Bousse, N. F. de Rooij and P. Bergveld, Operation of chemically sensitive fieldeffect sensors as a function of the insulator-electrolyte interface, IEEE Trans. Electron. Devices, ED-30 (1983) 1263 - 1270.

19 P. Bergveld, The development and application of FET-based biosensors, Biosensors, 2 (1986) 15 - 33 .

20 J. A. Voorthuyzen, H. van Vossen and P. Bergveld, Preparation and evaluation of tantalum pentoxide films for ISFET application, Proc. Eurosensors I, Cambridge, U.K., 1987 , pp. $43-44$.

21 P. Bergveld, A. van den Berg, P. D. van der Wal, M. Skowronska-Ptasonska, E. J. R. Sudhölter and D. N. Reinhoudt, How electrical and chemical requirements for REFETs may coincide, Sensors and Actuators, 18 (1989) in press.

22 B. H. van der Schoot and P. Bergveld, Coulometric sensors, the application of a sensor-actuator system for long-term stability in chemical sensing, Senbors and Actuators, 13 (1988) $251-262$.

23 B. H, van der Schoot and P. Bergveld, ISFET-based enzyme sensors, Biosensors, 3 (1987/1988) $161 \cdot 186$.

24 R. B. M. Schasfoort, P. Bergveld, J. Bomer, R. P. H. Kooyman and J. Greve, Modulation of the ISFET response by an immunological reaction, Sensors and Actuators, 17 (1989) 531.

25 A. J. Kölling, S. Koomen, P. Bergveld and E. Seevinck, Two-lead multiplex system for sensor array applications, Sensors and Actuators, 17 (1989) 623. 